\title{
Vessel Wall Enhancement on Black-Blood MRI Predicts Acute and Future Stroke in Cerebral Amyloid Angiopathy
}

\author{
(D).S. McNally, (D)A. Sakata, (D) M.D. Alexander, (D).D. Dewitt, (D).A. Sonnen, (D) S.T. Menacho, (D) G.J. Stoddard, (D) S.-E. Kim, and
} (iD) A.H. de Havenon

if 0 프

\begin{abstract}
BACKGROUND AND PURPOSE: Cerebral amyloid angiopathy (CAA) is a known risk factor for ischemic stroke though angiographic imaging is often negative. Our goal was to determine the relationship between vessel wall enhancement (VWE) in acute and future ischemic stroke in CAA patients.
\end{abstract}

MATERIALS AND METHODS: This was a retrospective study of patients with new-onset neurologic symptoms undergoing $3 T$ vessel wall MR imaging from 2015 to 2019. Vessel wall enhancement was detected on pre- and postcontrast flow-suppressed 3D T7WI. Interrater agreement was evaluated in cerebral amyloid angiopathy-positive and age-matched negative participants using a prevalence- and bias-adjusted kappa analysis. In patients with cerebral amyloid angiopathy, multivariable Poisson and Cox regression were used to determine the association of vessel wall enhancement with acute and future ischemic stroke, respectively, using backward elimination of confounders to $P<.20$.

RESULTS: Fifty patients with cerebral amyloid angiopathy underwent vessel wall MR imaging, including 35/50 (70.0\%) with ischemic stroke and $29 / 50$ (58.0\%) with vessel wall enhancement. Prevalence- and bias-corrected kappa was $0.82(95 \% \mathrm{Cl}, 0.71-0.93)$. The final regression model for acute ischemic stroke included vessel wall enhancement (prevalence ratio $=1.5 ; 95 \% \mathrm{Cl}, 1.1-2.2 ; P=.022$ ), age (prevalence ratio $=1.02 ; 95 \% \mathrm{Cl}, 1.0-1.05 ; P=.036$ ), time between symptoms and $\mathrm{MR}$ imaging (prevalence ratio $=0.9 ; 95 \% \mathrm{Cl}, 0.8-0.9 ; P<.001$ ), and smoking (prevalence ratio $=0.7 ; 95 \% \mathrm{Cl}, 0.5-1.0 ; P=.042)$ with c-statistic $=0.92(95 \% \mathrm{Cl}, 0.84-0.99)$. Future ischemic stroke incidence with cerebral amyloid angiopathy was $49.7 \%(95 \% \mathrm{Cl}, 34.5 \%-67.2 \%)$ per year over a total time at risk of 37.5 person-years. Vessel wall enhancement-positive patients with cerebral amyloid angiopathy demonstrated significantly shorter stroke-free survival with $63.9 \%(95 \% \mathrm{Cl}$, $43.2 \%-84.0 \%)$ versus $32.2 \%(95 \% \mathrm{Cl}, 14.4 \%-62.3 \%)$ ischemic strokes per year, chi-square $=4.9, P=.027$. The final model for future ischemic stroke had a c-statistic of 0.70 and included initial ischemic stroke (hazard ratio $=3.4 ; 95 \% \mathrm{Cl}, 1.0-12.0 ; P=.053$ ) and vessel wall enhancement (hazard ratio $=2.5 ; 95 \% \mathrm{Cl}, 0.9-7.0 ; P=.080$ ).

CONCLUSIONS: Vessel wall enhancement is associated with both acute and future stroke in patients with cerebral amyloid angiopathy.

ABBREVIATIONS: CAA = cerebral amyloid angiopathy; $\mathrm{HR}=$ hazard ratio; $P R=$ prevalence ratio; VWE = vessel wall enhancement; vwMRI = vessel wall MR imaging; AIS = acute ischemic stroke; SPACE = sampling perfection with application-optimized contrasts by using different flip angle evolutions

V essel wall enhancement (VWE) can be detected using vessel wall MR imaging (vwMRI) using flow-suppressed, contrastenhanced black-blood T1-weighted sequences. ${ }^{1,2}$ In the setting of

Received October 12, 2020; accepted after revision December 11

From the Department of Radiology (J.S.M., A.S., M.D.A., S.-E.K.), Utah Center for Advanced Imaging Research; Utah, and Departments of Pathology (J.A.S.),

Neurosurgery (S.T.M.), Internal Medicine (G.J.S.), and Neurology (L.D.D., A.H.d.H.), University of Utah, Salt Lake City, Utah

Gregory J. Stoddard performed statistical analyses.

This work was supported by the American Heart Association Scientist Development Grant 17SDG33460420 (McNally), NIH/NINDS K23NS105924 (A.H.H.), a grant from the National Institutes of Health R01 HL127582 (S.-E.K., J.S.M.). This investigation was supported by the University of Utah Population Health Research (PHR) Foundation, with funding in part from the National Center for Research Resources and the National Center for Advancing Translational Sciences, National Institutes of Health, through Grant ULITR002538 (G.J.S., formerly 5ULITR001067-05, 8ULITR000105 and ULIRR025764). intracranial atherosclerosis, VWE is a known independent risk factor for acute ischemic stroke (AIS). ${ }^{3,4}$ Other pathologies also affect the vessel wall, including vasculitis, reversible cerebral vasoconstriction syndrome, and Moyamoya disease and their findings on vwMRI that have been previously described. ${ }^{1,5,6}$ Very recently, a case series found VWE in 2 of 5 patients (40\%) with cerebral amyloid angiopathy (CAA). ${ }^{7}$ Although this small study showed

Please address correspondence to Scott McNally, MD, PhD, University of Utah, Department of Radiology, 30 North 1900 East \#1A071, Salt Lake City, UT 84132-2140; e-mail: scott.mcnally@hsc.utah.edu

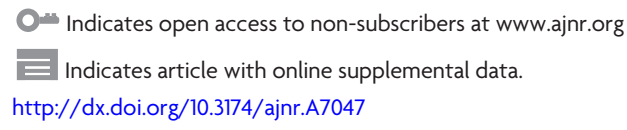


that VWE can occur in patients with noninflammatory CAA, its neurologic impact is unknown.

In addition to lobar hemorrhage, CAA is an important cause of transient neurologic complaints (amyloid spells), cognitive impairment, and ischemic infarcts. ${ }^{8-10}$ The pathogenesis of CAA is complex and related to amyloid- $\beta$ deposition in the small- to medium-sized vessel walls, resulting in necrosis, vessel rupture, or thrombosis. ${ }^{11-13}$ Because of this, imaging techniques that highlight vessel wall pathology, such as vwMRI, may have diagnostic and prognostic impact in patients with CAA. Brain imaging currently plays a vital role in CAA diagnosis using the modified Boston criteria. ${ }^{14}$ The most common acute imaging finding in patients with CAA is hemorrhage from vessel rupture. ${ }^{15}$ Microinfarcts can be seen in animal models of $\mathrm{CAA}^{16}$ and are present in $30 \%-60 \%$ of patients with $\mathrm{CAA},{ }^{17,18}$ contributing to cortical thinning. ${ }^{19} \mathrm{CAA}$ imaging criteria depend primarily on the presence of prior hemorrhage on susceptibility-weighted sequences, ${ }^{20}$ including siderosis and microhemorrhages in lobar, cortical- or subcortical locations. ${ }^{14}$ CAA disproportionately affects older adults, with increasing prevalence after age 60 years. $^{21}$ Because of this, the modified Boston criteria for CAA use a threshold of 55 years or older.

Patients with CAA often undergo work-up for acute neurologic deficits concerning for ischemic stroke, which can be detected on MR imaging. ${ }^{22}$ Evaluation of ischemic stroke risk in patients with CAA has important diagnostic and prognostic impact because it is a significant contributor to cognitive decline. ${ }^{23}$ These patients have complex medical histories, and because vessel wall pathology may not easily be seen with lumen imaging, this necessitates further evaluation with vessel wall imaging techniques. Because of their complicated nature, neurology consultation and vwMRI are often performed in the work-up of patients with CAA at our institution. In light of this and given the importance of VWE in a variety of intracranial vasculopathies, our goal was to determine the association of VWE with AIS in patients with CAA undergoing vwMRI during stroke work-up. In this study, we evaluated both acute concurrent and future ischemic stroke risk while controlling for potential cerebrovascular confounders. Our hypothesis was that in patients with CAA, VWE would be associated with both concurrent and future ischemic stroke.

\section{MATERIALS AND METHODS}

The data in this manuscript are available upon reasonable request.

\section{Study Design and Cohort}

Institutional review board approval was obtained before this retrospective study at our academic center from 2015 to 2019 in patients undergoing vwMRI for acute neurologic deficits concerning for ischemic stroke. Because of the retrospective nature, informed consent was not required by the institutional review board. In this protocol, all patients with CAA included had probable or possible CAA (based on modified Boston criteria and microhemorrhages detected before vwMRI) and documented acute focal neurologic deficits warranting vwMRI during the stroke work-up. All patients with CAA admitted under the neurology team with suspected stroke during this time period underwent vwMRI work-up per protocol. Although a few scans exhibited mild motion artifacts, none was sufficient to exclude any participants from interpretation. Chart review was used to determine age, sex, and other potential cerebrovascular confounders at the time of vwMRI.

\section{CAA Diagnosis}

Patients diagnosed with probable or possible CAA were included in this study. Modified Boston criteria were used by a vascular neurologist to diagnose CAA. ${ }^{14}$ Briefly, modified Boston criteria for probable CAA included age 55 years or older, appropriate clinical history, and MR imaging findings demonstrating either 1) multiple hemorrhages restricted to lobar, cortical, or corticosubcortical regions of varying sizes or ages without another cause or 2) a single lobar, cortical, or corticosubcortical hemorrhage and focal $(\leq 3$ sulci) or disseminated ( $>3$ sulci) cortical superficial siderosis without another cause. Cases of possible CAA were also included based on the modified Boston criteria definition: single lobar, cortical, or corticosubcortical hemorrhage without other cause. Twelve of our CAA cases were also confirmed with biopsy.

\section{vwMRI Protocol}

All MR imaging was obtained at 3T on Verio, Trio, or Prisma platforms (Siemens) with standard head coils and a standard contrast dose of MultiHance (Bracco), $0.1 \mathrm{mmol} / \mathrm{kg}$. Sequences included those in the Online Supplemental Data. The sampling perfection with application-optimized contrasts by using different flip angle evolutions (SPACE; Siemens) sequences were acquired with delay alternating with nutation for tailored excitation flow suppression. ${ }^{24}$ Images were obtained in this order-precontrast: DWI, SWI, TOF, T2 SPACE, T1 SPACE and postcontrast: TOF and T1 SPACE.

\section{Brain Parenchymal Findings}

Both acute and future ischemic stroke were determined using the American Heart Association definition of CNS infarction as previously described. ${ }^{25}$ Briefly, acute or future ischemic stroke was defined by brain cell death attributable to ischemia based on 1) imaging evidence of cerebral infarction or 2) clinical symptoms persisting $\geq 24$ hours, with other causes excluded. We also reviewed neurovascular clinic or inpatient charts to determine presence of AIS occurring at the time of imaging for concurrent stroke and at follow-up visits or neuroimaging for future stroke. Although all patients with CAA in this study had the clinical suspicion of stroke, only DWI-positive and low-ADC value acute infarcts were included as positive for AIS. In addition, the DWI positive infarct did not have to necessarily explain the patient's presentation and could be included even if clinically silent. The variable time between symptom onset and MR imaging was included as a potential confounder in the statistical analysis. Acute infarct was defined using DWI derived from DTI trace images described previously with parameters in the Online Supplemental Data. ${ }^{26}$ DTI trace outperforms conventional diffusion-weighted sequences in detecting acute infarcts. ${ }^{27}$ Brain DWI was interpreted by a subspecialty-trained, Certificate of Added Qualification-certified neuroradiologist blinded to vascular imaging. Follow-up imaging was only performed if patients developed an additional new, acute neurologic deficit concerning for ischemic stroke. DWI was the primary imaging determinant for both acute and future ischemic infarct in all patients. Although we had access to both CT and 
FLAIR imaging for all patients, these did not show additional interval infarcts not detected by DWI. Acute hemorrhage was also diagnosed on a CT scan by a neuroradiologist blinded to vascular imaging.

\section{Vessel Wall Enhancement}

Two separate board-certified radiologists evaluated VWE using T1 SPACE pre- and postcontrast sequences on vwMRI, blinded to additional imaging or clinical information. In cases of disagreement, a third radiologist was used as a tiebreaker. A signal threshold was used for VWE positivity using signal at or above the pituitary infundibulum as previously described. ${ }^{1,3}$ VWE was graded in a binary manner-either positive or negative for the entire set of brain images of each patient. This was done for the whole brain without regard to vascular segment, nor was it necessarily coupled with the area of DWI positivity. In addition to evaluating whole-brain VWE, we evaluated territorial VWE in patients with acute ischemic infarcts. VWE was evaluated in medium to small artery walls and only in vessels with confirmed arterial flow visible on TOF (including the first- through fourth-order branches of the circle of Willis). The TOF was only used to confirm the vessel was an artery and not a vein, and the VWE readers did not simultaneously grade the TOF for stenosis (see later discussion). Enhancement along a single vessel wall was considered positive, though in all positive patients, $>1$ vessel was involved.

\section{Stenosis on VwMRI}

Maximum percent diameter stenosis was determined per the Warfarin-Aspirin Symptomatic Intracranial Disease trial measurement of intracranial stenosis. ${ }^{28}$ The subspecialty-trained, Certificate of Added Qualification-certified neuroradiologist determined stenosis blinded from DWI and VWE images at the time of TOF evaluation (and performed after a 2-month washout period). Of all intracranial arteries visible on TOF, the artery with maximum stenosis was selected using a combination of axial imaging, multiplanar reformats, maximum intensity projections, and 3D reformats. Then the diameter (b) at the level of maximal stenosis and diameter (a) of the downstream vessel distal to the stenosis were used to calculate percent diameter stenosis using the formula $[(a-b) / a] \times$ $100 \%$. Stenosis was measured at the narrowest segment of the vessel (b) perpendicular to the long axis of the vessel on multiplanar reformats using a submillimeter measurement tool. From the WarfarinAspirin Symptomatic Intracranial Disease trial, in cases in which the downstream segment of the vessel was diseased, a proximal normal vessel segment was used for the comparison diameter (a). In cases in which the entire vessel was diseased, the contralateral vessel was used for the comparison diameter (a).

\section{Interrater Reliability}

For interrater reliability, we evaluated an equal number of negative control participants alongside the patients with CAA. This group of CAA-negative patients were age-matched (older than 55 years old) and had undergone vwMRI during the same time period, with the same protocol, and in the same scanners. These patients had no evidence of probable or possible CAA, vascular stenosis, aneurysm, or acute stroke or hemorrhage. Interrater reliability for the binary vwMRI measurement of positive or negative enhancement was calculated in the combined group of CAA and negative control participants blinded to all other imaging and clinical covariates using prevalence- and bias-adjusted kappa statistics.

\section{Statistical Analysis}

Univariable binary Poisson regression, with robust standard errors, was used in the patients with CAA to determine the prevalence ratio (PR) of vwMRI factors, as well as clinical confounders for concurrent AIS as determined by retrospective chart review. These confounders included maximum percent stenosis, age, female sex, hypertension, hyperlipidemia, diabetes, smoking, antiplatelet use, anticoagulation use, antihypertension use, and statin use. Systemic inflammatory markers (erythrocyte sedimentation rate and C-reactive protein) were also recorded in 30 of the 50 patients with CAA. The time between symptom onset and MR imaging was also evaluated in relation to AIS. Next, all potential confounding variables with $P<.20$ from the univariable analysis were placed in an initial multivariable binary Poisson regression model with robust standard errors for the prediction of AIS; then variables were eliminated in a backward fashion until all remaining variables met the threshold $P<.20$.

Accuracy of the prediction of AIS was determined using the area under the receiver operating characteristic curve, also called the c-statistic. Lastly, we investigated whether the presence of VWE predicted future ischemic stroke using Kaplan-Meier survival analysis to estimate the ischemic event rate. Ischemic event rates per 100 person-years were calculated for each outcome, and the formula annual risk $=1(\exp [-$ event rate $\times$ time $])$ was used to estimate the absolute annual risk. Time to event was further analyzed for stroke by use of univariable followed by multivariable Cox regression analysis. After univariable analysis, factors with $P<.20$ were placed in an initial multivariable model; then variables underwent backward elimination to a threshold $P<.20$. All patients were followed up by chart review for interval stroke symptoms to the end of the study in 2019. Cox regression was used to account for variable times to event.

In binary outcome models, 5 outcome events for every predictor are sufficient to avoid overfitting. ${ }^{29}$ Our final model for future stroke met this. The final Poisson regression model, however, did not, and this is known to increase the likelihood of finding falsepositive predictors. ${ }^{30}$ To assess the stability of the final Poisson model, the bootstrap inclusion fraction was computed for each predictor, which is the percentage of times the variable remains in the final model in a large number of bootstrap resamples in which the variable selection is repeated. ${ }^{31,32}$ Predictors with bootstrap inclusion fractions $<50 \%$ were dropped from the final model as unreliable because these would not likely remain as significant predictors in future datasets.

All statistical analyses were performed using Stata-15.1 statistical software (StataCorp).

\section{RESULTS}

\section{CAA Study Population}

Characteristics of the patients with CAA are listed in Table 1. A total of 50 patients met CAA study criteria, with vwMRI obtained within 7 days of stroke symptom onset. These patients included those with probable (43/50) and possible (7/50) CAA; 35/50 
(70.0\%) patients had AIS, 23/50 (46.0\%) had acute hemorrhage, and $19 / 50(38.0 \%)$ had both. Potential confounders included maximum percent stenosis $(14.9 \pm 20.8$, mean \pm SD), patient age $(71.9 \pm 7.7$ years), female sex $(27 / 50,54.0 \%)$, hypertension (43/50, 86.0\%), hyperlipidemia $(32 / 50,64.0 \%)$, diabetes $(14 / 50$, $28.0 \%)$, smoking (19/50, 38.0\%), antiplatelet use (18/50, 36.0\%), anticoagulant use $(5 / 50,10.0 \%)$, antihypertensive use $(37 / 50$, $74.0 \%)$, statin use $(27 / 50,54.0 \%)$, time between symptom onset and MR imaging (3.9 \pm 2.6 days), and elevated inflammatory markers, which were recorded in 30 patients (10, 33.3\%).

\section{VWE Interrater Reliability}

The 50 patients with CAA were combined with 50 negative control participants without CAA, acute hemorrhage, ischemic stroke, or vascular pathology. Patients with CAA demonstrated VWE (29/50, $58.0 \%)$, whereas negative control participants did not (0/50, 0.0\%). Fig 1 illustrates 3 separate patients with CAA with variable degrees

\section{Table 1: Characteristics of patients with CAA}

\begin{tabular}{lc}
\hline \multicolumn{1}{c}{ Patient Characteristic } & $\boldsymbol{n}=50$ \\
\hline Probable CAA, $n(\%)$ & $43(86.0)$ \\
Biopsy proved, $n(\%)$ & $12(24.0)$ \\
Possible CAA, $n(\%)$ & $7(14.0)$ \\
Neurologic outcomes & \\
Ischemic stroke, $n(\%)$ & $35(70.0)$ \\
Hemorrhage, $n(\%)$ & $23(46.0)$ \\
Both, $n$ (\%) & $19(38.0)$ \\
VWE & $29(58.0)$ \\
Clinical confounders & \\
Maximum percent stenosis, mean (SD) & $14.9(20.8)$ \\
Age, mean (SD), y & $71.9(7.7)$ \\
Female sex, $n(\%)$ & $27(54.0)$ \\
Hypertension, $n(\%)$ & $43(86.0)$ \\
Hyperlipidemia, $n(\%)$ & $32(64.0)$ \\
Diabetes, $n(\%)$ & $14(28.0)$ \\
Smoking, $n(\%)$ & $19(38.0)$ \\
Antiplatelet, $n$ (\%) & $18(36.0)$ \\
Anticoagulation, $n(\%)$ & $5(10.0)$ \\
Antihypertension, $n$ (\%) & $37(74.0)$ \\
Statin, $n$ (\%) & $27(54.0)$ \\
Time between symptom onset and MR imaging in & $3.9(2.6)$ \\
$\quad$ days, mean (SD) & \\
Inflammatory marker elevation, $n$ (\%), $n=30$ available & $10(33.3)$ \\
\hline
\end{tabular}

of VWE including a VWE negative scan, a single VWE positive artery on a single image, and a VWE positive scan with multiple arteries involved on the same image. In the combined group of 50 CAA and 50 negative control patients, the radiology reviewers agreed 91/100 times with a third tie-breaker used 9 times. Interrater reliability for VWE was "substantial," with a prevalenceand-bias-corrected kappa $=0.82(95 \%$ CI, 0.71-0.93).

\section{Factors Associated with Acute Ischemic Stroke in Patients with CAA}

A representative patient with CAA presenting with AIS and VWE is shown in Fig 2. Leptomeningeal and brain biopsy was also performed in this particular patient as shown in the Online Supplemental Data. In this index case, pathology showed evidence of CAA coupled with vessel wall inflammation. Overall, 35/50 patients with CAA were positive for AIS, and 23 of these were VWE positive, with 22/23 matched to the vascular territory (95.7\%). There were $5 / 50$ patients with CAA with $>50 \%$ stenosis and $0 / 50$ with $>70 \%$ stenosis. Of those with $>50 \%$ stenosis, $1 / 5$ had an acute ischemic infarct within the stenosis territory, though this patient did not have a future ischemic stroke. Univariable analysis with factors and potential confounders associated with AIS are depicted in Table 2.

Factors associated with concurrent AIS meeting the $P<.20$ threshold were entered into the initial multivariable model. These included: $\mathrm{VWE}(\mathrm{PR}=1.6 ; 95 \% \mathrm{CI}, 1.0-2.5 ; P=.044)$, age $(\mathrm{PR}=$ 1.01; 95\% CI, 0.99-1.05; $P=.146$ ), diabetes ( $\mathrm{PR}=1.3$; 95\% CI, $1.0-1.9 ; P=.080)$, time between symptom onset and $\mathrm{MR}$ imaging $(\mathrm{PR}=0.9 ; 95 \% \mathrm{CI}, 0.8-1.0 ; P=.006)$ and smoking $(\mathrm{PR}=0.7$; 95\% CI, 0.4-1.1; $P=.095)$. These did not include maximum percent stenosis ( $\mathrm{PR}=1.6$; 95\% CI, $0.7-3.6 ; P=.297$ ) or other confounders. Also, in the 30 patients with recorded systemic inflammatory markers, these were not associated with AIS (PR = 1.2; 95\% CI, 0.9-1.7; $P=.282$ ). After sequential backwards elimination to a threshold $P<.20,4$ factors remained in the final model: VWE (PR $=1.5 ; 95 \% \mathrm{CI}, 1.1-2.2 ; P=.022)$, age ( $\mathrm{PR}=1.02 ; 95 \%$ CI, 1.0-1.05; $P=.036)$, time between symptoms and MR imaging $(\mathrm{PR}=0.9 ; 95 \% \mathrm{CI}, 0.8-0.9 ; P<.001)$, and smoking $(\mathrm{PR}=0.7$; $95 \%$ CI, $0.5-1.0 ; P=.042)$. The final model discriminatory value
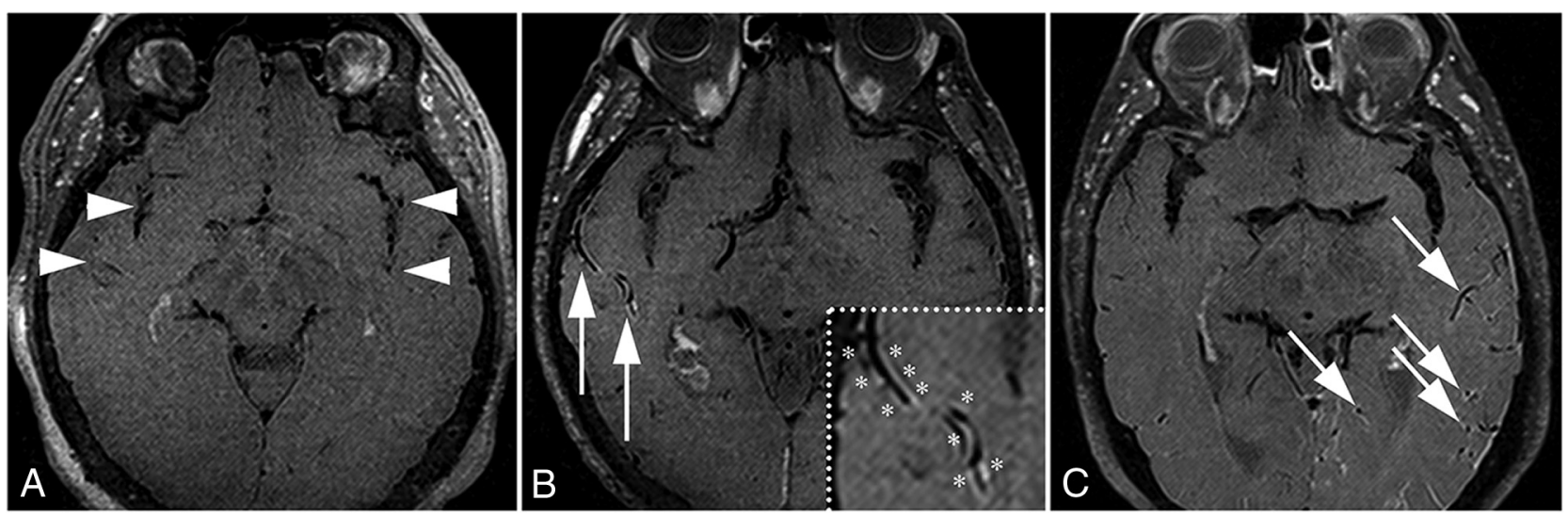

FIG 1. Variation of VWE findings in patients with CAA. A, This patient with CAA had no VWE along the intracranial arteries (arrowheads). B, There was VWE along a single right MCA branch in the representative image (arrows, inset with asterisks). $C$, There were multiple left MCA branches with VWE on the same image (arrows). 

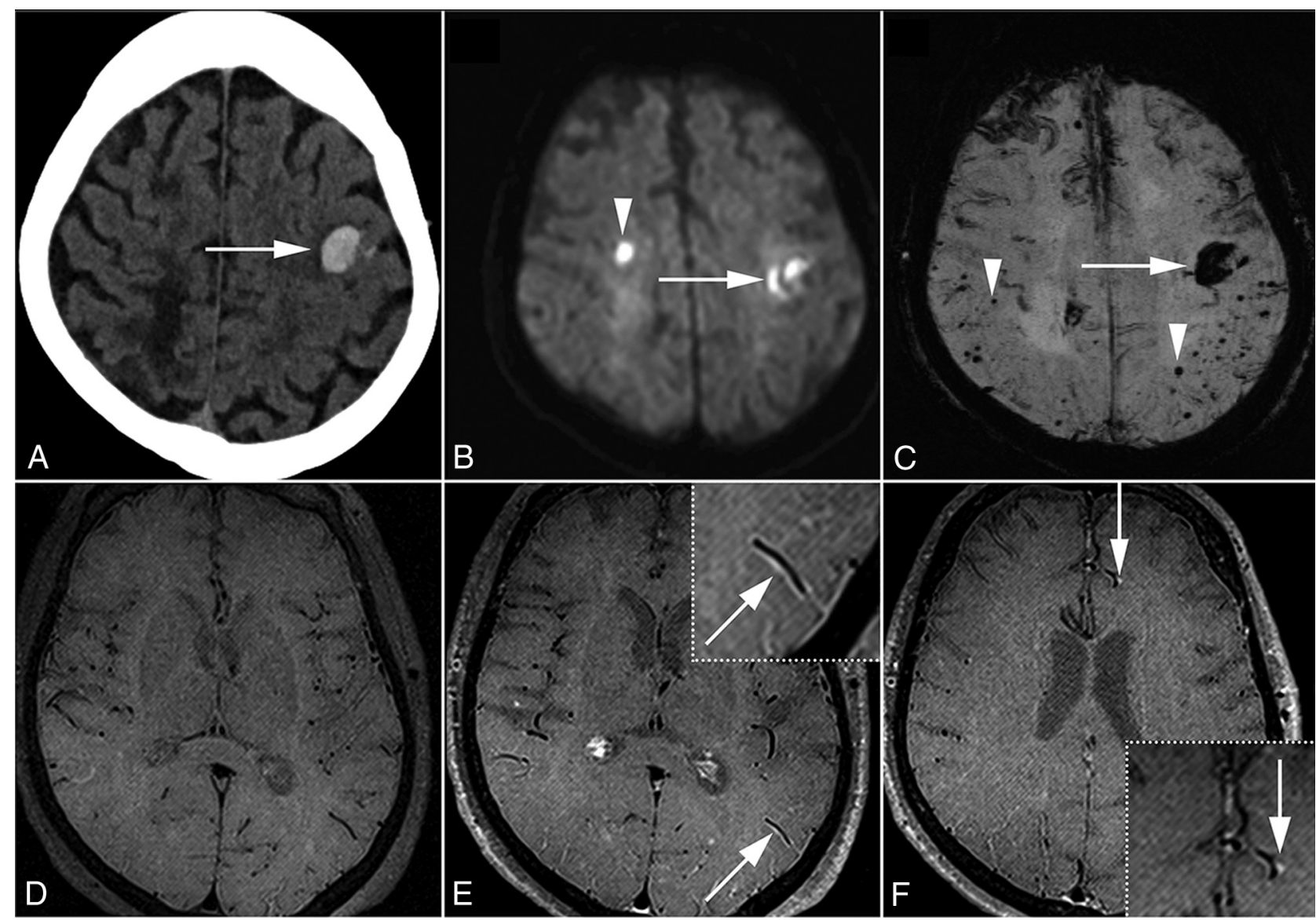

FIG 2. Representative patient with CAA with acute hemorrhage, AIS, and VWE. This 69-year-old woman with acute right hand incoordination and aphasia. Acute left frontal parenchymal hemorrhage was detected on CT (A, arrow). DWI showed a small acute right frontal infarct (B, arrowhead) in addition to the hemorrhage (arrow). SWI demonstrated multiple peripheral and subcortical microhemorrhages (C, arrowheads) in addition to the acute hemorrhage (arrow). Vessel wall imaging with pre- $(D)$ and postcontrast T1 SPACE (E and F) revealed VWE along arterial branches ( $E$, arrow, inset; $F$, arrow, inset).

Table 2: Univariable Poisson regression CAA vwMRI findings and AIS

\begin{tabular}{lcccc}
\hline \multicolumn{1}{c}{ Stroke Predictor } & $\begin{array}{c}\text { Stroke (-) } \\
(\boldsymbol{n}=15)\end{array}$ & $\begin{array}{c}\text { Stroke (+) } \\
(\boldsymbol{n}=35)\end{array}$ & $\begin{array}{c}\text { Univariable } \\
\text { PR (95\% CI) }\end{array}$ & $\begin{array}{c}\boldsymbol{P} \\
\text { Value }\end{array}$ \\
\hline VWE, $n(\%)$ & $5(33.3)$ & $24(68.6)$ & $1.6(1.0-2.5)$ & .044 \\
Maximum percent stenosis, mean (SD) & $10.3(20.7)$ & $16.9(20.8)$ & $1.6(0.7-3.6)$ & .297 \\
Age, mean (SD) & $69.4(8.5)$ & $73.0(7.2)$ & $1.01(0.99-1.05)$ & .146 \\
Female, $n(\%)$ & $7(46.7)$ & $20(57.1)$ & $1.1(0.8-1.7)$ & .507 \\
Hypertension, $n(\%)$ & $13(86.7)$ & $30(85.7)$ & $1.0(0.6-1.6)$ & .928 \\
Hyperlipidemia, $n$ (\%) & $10(66.7)$ & $22(62.9)$ & $1.0(0.7-1.4)$ & .796 \\
Diabetes, $n(\%)$ & $2(13.3)$ & $12(34.3)$ & $1.3(1.0-1.9)$ & .080 \\
Smoking, $n(\%)$ & $9(60.0)$ & $11(31.4)$ & $0.7(0.4-1.1)$ & .095 \\
Antiplatelet, $n(\%)$ & $5(33.3)$ & $13(37.1)$ & $1.1(0.7-1.5)$ & .796 \\
Anticoagulation, $n(\%)$ & $1(6.7)$ & $4(1.4)$ & $1.2(0.7-1.9)$ & .546 \\
Antihypertension, $n$ (\%) & $12(80.0)$ & $25(71.4)$ & $0.9(0.6-1.3)$ & .499 \\
Statin, $n$ (\%) & $8(53.3)$ & $19(54.3)$ & $1.0(0.7-1.5)$ & .951 \\
Time between symptom onset and MR & $5.5(2.4)$ & $3.2(2.3)$ & $0.9(0.8-1.0)$ & .006 \\
$\quad$ imaging in days, mean (SD) & & & & \\
Inflammatory marker elevation, $n(\%)$, & $1(16.7)$ & $9(37.5)$ & $1.2(0.9-1.7)$ & .282 \\
$\quad n=30$ available & $n=6$ & $n=24$ & & \\
\hline
\end{tabular}

Note:-Univariable Poisson regression was initially performed with PR values, with $95 \% \mathrm{Cl}$ and $P$ values above. All potential confounding variables with $P<.20$ from the univariable analysis were placed in an initial multivariable Poisson regression model for AIS; then variables were eliminated in a backward fashion to the threshold $P<.20$. Four factors remained in the final multivariable regression model for AIS: VWE (PR $=1.5 ; 95 \% \mathrm{Cl}, 1.1-2.2 ; P=.022)$, age $(\mathrm{PR}=1.02 ; 95 \% \mathrm{Cl}, 1.0-1.05 ; P=.036)$, time between symptoms and $\mathrm{MR}$ imaging $(\mathrm{PR}=0.9 ; 95 \% \mathrm{Cl}, 0.8-0.9$; $P<.001)$, and smoking ( $\mathrm{PR}=0.7 ; 95 \% \mathrm{Cl}, 0.5-1.0 ; P=.042)$ was c-statistic $=0.92 \quad(95 \%$ CI, $0.84-$ 0.99). The bootstrap inclusion fraction was computed for each predictor, and all variables in the final model were stable, meaning the results were not due to overfitting.

\section{Future Stroke Prediction}

In this study patients with CAA had an overall time at risk of 37.5 personyears and incident stroke event rate of $49.7 \%$ (95\% CI, $34.5 \%-67.2 \%$ ) per year. Kaplan-Meier survival analysis (Fig 3) demonstrated significantly shorter stroke-free survival with VWE. VWE-positive patients with CAA had a higher ischemic event rate of $63.9 \%$ (95\% CI, $43.2 \%-84.0 \%$ ) per year compared with VWE-negative patients with CAA with $32.2 \%$ (95\% CI, 14.4\%-62.3\%) per year, with chi-square $=4.9$ and $P=.027$. Univariable Cox regression analysis for 
stroke showed that 3 factors significantly increased future stroke risk: initial stroke (hazard ratio $[\mathrm{HR}]=4.2 ; 95 \% \mathrm{CI}, 1.2-14.3$; $P=.023)$, VWE $(\mathrm{HR}=3.1 ; 95 \% \mathrm{CI}, 1.1-8.6 ; P=.027)$, and

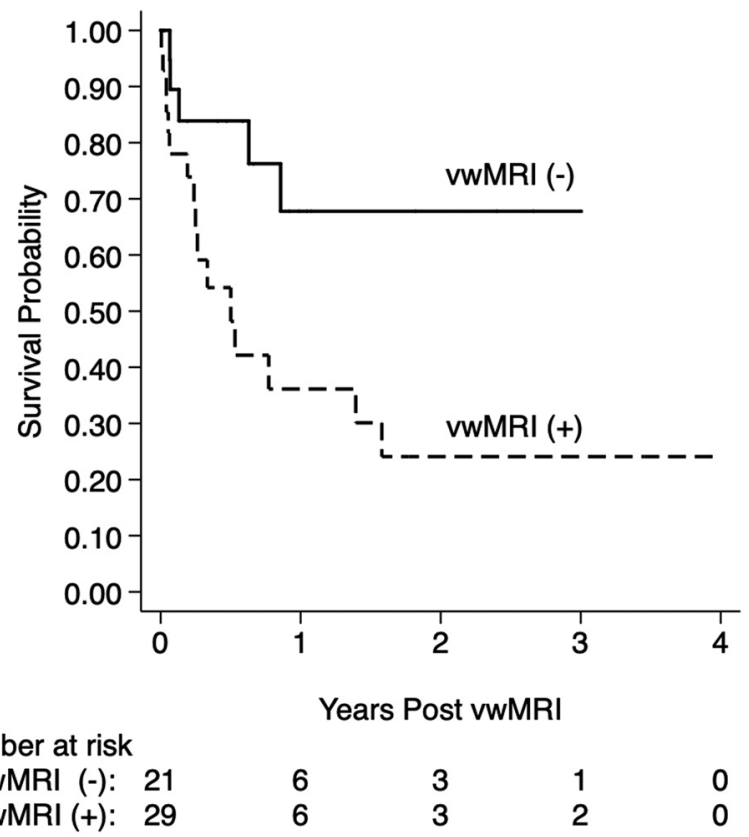

FIG 3. Future stroke risk analysis. Kaplan-Meier survival curves are shown for patients with CAA with versus without VWE, vwMRI (+) versus (-). In the 50 participants, the total time at risk was 37.5 personyears, and incidence event rate of ischemic stroke was $49.7 \%(95 \% \mathrm{Cl}$, $34.5 \%-67.2 \%)$ per year. Patients with CAA with VWE demonstrated significantly shorter stroke-free survival with an event rate of $63.9 \%$ (95\% Cl, 43.2\%-84.0\%) per year compared with $32.2 \%(95 \% \mathrm{Cl}, 14.4 \%-$ $62.3 \%$ ), chi-square $=4.9, P=.027$. As discussed in Table 3 , multivariable Cox regression followed by backward elimination to a threshold $P$ value of 0.20 showed that VWE was 1 of 2 factors remaining in the final future stroke prediction model (VWE HR $=2.5 ; 95 \% \mathrm{Cl}, 0.9-7.0 ; P=.080$ and initial ischemic stroke $\mathrm{HR}=3.4 ; 95 \% \mathrm{Cl}, 1.0-12.0 ; P=.053$ ). smoking $(\mathrm{HR}=0.5 ; 95 \% \mathrm{CI}, 0.2-1.3 ; P=.135)$. These were the only variables with $P<.20$ from the univariable analysis and were placed in the initial multivariable Cox regression model. After multivariable regression, only 2 variables remained in the final model at the $P<.20$ threshold and predicted future ischemic stroke with a c-statistic of 0.70 . The final model consisted of initial ischemic stroke ( $\mathrm{HR}=3.4 ; 95 \% \mathrm{CI}, 1.0-12.0 ; P=.053)$ and VWE $(\mathrm{HR}=2.5 ; 95 \% \mathrm{CI}, 0.9-7.0 ; P=.080)$.

\section{DISCUSSION}

This study adds support to the growing literature on ischemic stroke risk in CAA by investigating the prognostic impact of vwMRI. Our data show that vwMRI can identify VWE in patients with CAA with high interrater reliability, paralleling previous studies in other vasculopathies. ${ }^{1,3}$ Furthermore, VWE was an essential predictor of both acute and future ischemic stroke in CAA, with VWE associated with significantly shorter stroke-free survival.

Ischemic stroke occurs often in patients with CAA despite negative angiographic imaging. ${ }^{33,34}$ Our study confirms the lack of association with lumen stenosis. This corresponds to prior work finding no association between vascular occlusion or stenosis with white matter hyperintensities in CAA. ${ }^{35,36}$ Although lumen imaging lacks the resolution of small-vessel pathology, this limitation does not appear to affect contrast leakage as detected by vwMRI. Our observed $70 \%$ prevalence for concurrent ischemic infarcts and $42 \%$ prevalence of future stroke is slightly higher than previously reported acute microinfarcts in $30 \%-60 \%$ of patients with CAA. ${ }^{17,18}$ This may reflect some degree of selection bias, given that only symptomatic patients with CAA underwent vwMRI. Because of this, these data should be applied to symptomatic patients with CAA presenting with acute neurologic deficits. Variable time between stroke symptom onset and vwMRI was a potential limitation, though this was accounted for in the final regression model and VWE remained associated with AIS. Other potential limitations include variable follow-up methods and time periods for stroke follow-up evaluation.

Table 3: Univariable Cox regression for future ischemic stroke

\begin{tabular}{|c|c|c|c|c|}
\hline Stroke Predictor & Future Stroke $(-)(n=29)$ & Future Stroke $(+)(n=21)$ & Univariable HR $(95 \% \mathrm{Cl})$ & $P$ Value \\
\hline Initial stroke, $n(\%)$ & $17(58.6)$ & $18(85.7)$ & $4.2(1.2-14.3)$ & .023 \\
\hline VWE, $n(\%)$ & $13(44.8)$ & $16(76.2)$ & $3.1(1.1-8.6)$ & .027 \\
\hline Maximum percent stenosis, mean (SD) & $17.1(22.5)$ & $11.9(18.3)$ & $0.7(0.1-6.4)$ & .723 \\
\hline Age, mean (SD) & $72.6(8.2)$ & $71.0(7.1)$ & $1.0(0.9-1.0)$ & .651 \\
\hline Female, $n(\%)$ & $13(44.8)$ & $14(66.7)$ & $1.5(0.6-3.7)$ & .397 \\
\hline Hypertension, $n(\%)$ & $24(82.8)$ & $18(90.5)$ & $1.7(0.4-7.1)$ & .499 \\
\hline Hyperlipidemia, $n(\%)$ & $17(58.6)$ & $15(71.4)$ & $1.3(0.5-3.5)$ & .552 \\
\hline Diabetes, $n(\%)$ & $7(24.1)$ & $7(33.3)$ & $1.3(0.5-3.3)$ & .571 \\
\hline Smoking, $n(\%)$ & $14(48.3)$ & $6(28.6)$ & $0.5(0.2-1.3)$ & .135 \\
\hline (interval) Antiplatelet, $n(\%)$ & $9(31.0)$ & $6(28.6)$ & $0.8(0.3-2.1)$ & .649 \\
\hline (interval) Anticoagulation, $n(\%)$ & $3(10.3)$ & $1(4.8)$ & $0.4(0.05-2.8)$ & .336 \\
\hline (interval) Antihypertension, $n(\%)$ & $23(79.3)$ & $17(81.0)$ & $1.3(0.4-3.8)$ & .662 \\
\hline (interval) Statin, $n(\%)$ & $22(75.9)$ & $14(66.7)$ & $0.7(0.3-1.6)$ & .372 \\
\hline (interval) Immunosuppression, $n$ (\%) & $7(24.1)$ & $10(47.6)$ & $1.5(0.7-3.7)$ & .322 \\
\hline $\begin{array}{l}\text { Elevated inflammatory markers, } n(\%) \\
\qquad n=30 \text { available }\end{array}$ & $5(33.3) n=15$ & $5(33.3) n=15$ & $1.1(0.4-3.1)$ & .928 \\
\hline
\end{tabular}

Note:-Interval indicates interval treatment with the listed medication as a potential confounder.

Univariable Cox regression was initially performed with $\mathrm{HR}$ values, with $95 \% \mathrm{Cl}$ and $P$ values below. All potential confounding variables with $P<.20$ from the univariable analysis were placed in an initial multivariable Cox regression model for future ischemic stroke; then variables were eliminated in a backward fashion to the threshold $P<.20$. Two factors remained in the final multivariable regression model for future ischemic stroke: initial ischemic stroke ( $\mathrm{HR}=3.4 ; 95 \% \mathrm{Cl}, 1.0-12.0 ; P=.053)$ and $\mathrm{VWE}$ $(\mathrm{HR}=2.5 ; 95 \% \mathrm{Cl}, 0.9-7.0 ; P=.080)$ 
Another limitation was the single-center nature, though multiple different scanners and separate observers were used for internal validation. With the growing clinical use of vwMRI it should soon be possible to confirm these findings at additional centers, potentially with future multicenter studies. Additionally, our study was not designed to determine best treatment or preventative strategies against future ischemic stroke in CAA. Although some additional confounders were associated with initial ischemic stroke, future stroke prevention is the critical issue. Also, although our study did not include asymptomatic CAA control participants, the patients with CAA without acute or follow-up infarcts on DWI served as the comparator groups in our regression analyses. A future study on asymptomatic patients with CAA could determine silent stroke risk in this population.

Our dataset also had limited information on VWE linked with CAA-related inflammation, which is characterized by rapid cognitive decline, vascular inflammation, and lobar edema. ${ }^{37,38}$ Systemic inflammation did not contribute to stroke risk in our group of patients with CAA, though the role of local inflammation is not yet well defined. Although most patients did not undergo biopsy, 1 patient in the Online Supplemental Data showed CAA-related inflammation on pathology that corresponded with VWE. It is clear though that VWE does not always meet the threshold of pathology-defined CAA-related inflammation related to leukocyte infiltration. ${ }^{7}$ Local proinflammatory cascades may still be activated, resulting in endothelial dysfunction and contrast leakage before overt leukocyte infiltration on pathology. The traditional dichotomy of CAA with or without inflammation may instead represent a spectrum of proinflammatory cascades as with other neuroimmunologic diseases, including multiple sclerosis. ${ }^{39}$ Without an overt link to inflammation on pathology, however, the use of novel immunotherapies in patients with CAA with VWE remains premature.

The strengths of this imaging study include a sizable number of patients with CAA undergoing unique evaluation with vwMRI. Although the retrospective design is a limitation, this applies to most imaging studies of patients with CAA. More important, our conclusions are relevant to symptomatic patients with CAA in whom diagnostic and treatment decisions are made on an urgent basis, and there was a high risk of future stroke correlated with VWE on initial vwMRI, which allows for prognostication in these patients. Also, despite the minority of patients undergoing confirmatory biopsy, the results remain generalizable to patients with suspected CAA by imaging. By evaluating all participants with CAA who underwent imaging, these results translate to clinical practice in the work-up of acute neurologic deficits or rapid neurologic decline. These patients with CAA tend to benefit the most from rapid diagnosis, stroke prevention strategies, and close follow-up. Future research is warranted to develop or identify treatment strategies against the CAA disease processes leading to VWE.

\section{CONCLUSIONS}

VWE can be reliably detected on black-blood vwMRI in patients with CAA presenting with new-onset focal neurologic symptoms, and this is associated with AIS. VWE was highly associated with concurrent AIS on multivariable regression analysis. Most important, initial stroke and VWE were essential factors predicting future ischemic stroke risk in this cohort of patients with CAA. The use of vwMRI can have diagnostic and prognostic impact in this high-risk population and may serve as the target for future trials aimed at decreasing stroke risk.

Disclosures: J. Scott McNally-RELATED: Grant: AHA, NIH, Comments: This work was supported by the American Heart Association Scientist Development Grant 17SDG33460420 (McNally), NIH/NINDS K23NS105924 (A.H.H.), a grant from the National Institutes of Health R01 HL127582 (S.-E.K., J.S.M.), and a grant from the National Institutes of Health, ULITR002538 (G.J.S.)*. Gregory StoddardUNRELATED: Grants/Grants Pending: NIH, Comments: A grant supports the statistical core I worked for, but it is not specific to this project. The NIH has requested we mention their grant numbers as an acknowledgment in the manuscript when I work on a project: This investigation was supported by the University of Utah Population Health Research (PHR) Foundation, with funding in part from the National Center for Research Resources and the National Center for Advancing Translational Sciences, National Institutes of Health, through Grant ULITR002538 (formerly 5ULITR001067-05, 8ULITR000105 and ULIRR025764)*. Adam de Havenon-UNRELATED: Grants/Grants Pending: NIH/ NINDS.* *Money paid to institution.

\section{REFERENCES}

1. Mossa-Basha M, Hwang WD, De Havenon A, et al. Multicontrast high-resolution vessel wall magnetic resonance imaging and its value in differentiating intracranial vasculopathic processes. Stroke 2015;46:1567-73 CrossRef Medline

2. Alexander MD, de Havenon A, Kim SE, et al. Assessment of quantitative methods for enhancement measurement on vessel wall magnetic resonance imaging evaluation of intracranial atherosclerosis. Neuroradiology 2019;61:643-50 CrossRef Medline

3. de Havenon A, Mossa-Basha M, Shah L, et al. High-resolution vessel wall MRI for the evaluation of intracranial atherosclerotic disease. Neuroradiology 2017;59:1193-1202 CrossRef Medline

4. Kim JM, Jung $\mathrm{KH}$, Sohn $\mathrm{CH}$, et al. Intracranial plaque enhancement from high resolution vessel wall magnetic resonance imaging predicts stroke recurrence. Int J Stroke 2016;11:171-79 CrossRef Medline

5. Mossa-Basha M, de Havenon A, Becker KJ, et al. Added value of vessel wall magnetic resonance imaging in the differentiation of Moyamoya vasculopathies in a non-Asian cohort. Stroke 2016;47:1782-88 CrossRef Medline

6. Mossa-Basha M, Alexander M, Gaddikeri S, et al. Vessel wall imaging for intracranial vascular disease evaluation. J Neurointerv Surg 2016;8:1154-59 CrossRef Medline

7. Hao Q, Tsankova NM, Shoirah H, et al. Vessel wall MRI enhancement in noninflammatory cerebral amyloid angiopathy. AJNR Am J Neuroradiol 2020;41:446-48 CrossRef Medline

8. Kimberly WT, Gilson A, Rost NS, et al. Silent ischemic infarcts are associated with hemorrhage burden in cerebral amyloid angiopathy. Neurology 2009;72:1230-35 CrossRef Medline

9. Gregoire SM, Charidimou A, Gadapa N, et al. Acute ischaemic brain lesions in intracerebral haemorrhage: multicentre cross-sectional magnetic resonance imaging study. Brain 2011;134:2376-86 CrossRef Medline

10. Vallat W, Maundrell A, Leyden J, et al. Cerebral amyloid angiopathy causing cortical microinfarction. J Clin Neurosci 2013;20:1802-04 CrossRef Medline

11. Mandybur TI. Cerebral amyloid angiopathy: the vascular pathology and complications. J Neuropathol Exp Neurol 1986;45:79-90 Medline

12. Vonsattel JP, Myers RH, Hedley-Whyte ET, et al. Cerebral amyloid angiopathy without and with cerebral hemorrhages: a comparative histological study. Ann Neurol 1991;30:637-49 CrossRef Medline

13. Yamada M. Cerebral amyloid angiopathy: emerging concepts. $J$ Stroke 2015;17:17-30 CrossRef Medline

14. Linn J, Halpin A, Demaerel P, et al. Prevalence of superficial siderosis in patients with cerebral amyloid angiopathy. Neurology 2010;74:1346-50 CrossRef Medline 
15. Smith EE, Eichler F. Cerebral amyloid angiopathy and lobar intracerebral hemorrhage. Arch Neurol 2006;63:148-51 CrossRef Medline

16. Milner E, Zhou ML, Johnson AW, et al. Cerebral amyloid angiopathy increases susceptibility to infarction after focal cerebral ischemia in Tg2576 mice. Stroke 2014;45:3064-69 CrossRef Medline

17. van Veluw SJ, Charidimou A, van der Kouwe AJ, et al. Microbleed and microinfarct detection in amyloid angiopathy: a high-resolution MRI-histopathology study. Brain 2016;139:3151-62 CrossRef Medline

18. Lauer A, van Veluw SJ, William CM, et al. Microbleeds on MRI are associated with microinfarcts on autopsy in cerebral amyloid angiopathy. Neurology 2016;87:1488-92 CrossRef Medline

19. Fotiadis P, van Rooden S, van der Grond J, et al. Cortical atrophy in patients with cerebral amyloid angiopathy: a case-control study. Lancet Neurol 2016;15:811-19 CrossRef Medline

20. Cheng AL, Batool S, McCreary CR, et al. Susceptibility-weighted imaging is more reliable than $\mathrm{T}^{\star}$-weighted gradient-recalled echo MRI for detecting microbleeds. Stroke 2013;44:2782-86 CrossRef Medline

21. Greenberg SM, Vonsattel JP. Diagnosis of cerebral amyloid angiopathy. Sensitivity and specificity of cortical biopsy. Stroke 1997;28:141822 CrossRef Medline

22. Ter Telgte A, Scherlek AA, Reijmer YD, et al. Histopathology of diffusion-weighted imaging-positive lesions in cerebral amyloid angiopathy. Acta Neuropathol 2020;139:799-812 CrossRef Medline

23. Hecht M, Kramer LM, von Arnim CAF, et al. Capillary cerebral amyloid angiopathy in Alzheimer's disease: association with allocortical/hippocampal microinfarcts and cognitive decline. Acta Neuropathol 2018;135:681-94 CrossRef Medline

24. Li L, Miller KL, Jezzard P. DANTE-prepared pulse trains: a novel approach to motion-sensitized and motion-suppressed quantitative magnetic resonance imaging. Magn Reson Med 2012;68:142338 CrossRef Medline

25. Sacco RL, Kasner SE, Broderick JP, et al. An updated definition of stroke for the 21st century: a statement for healthcare professionals from the American Heart Association/American Stroke Association. Stroke 2013;44:2064-89 CrossRef Medline

26. McNally JS, Kim SE, Yoon HC, et al. Carotid magnetization-prepared rapid acquisition with gradient-echo signal is associated with acute territorial cerebral ischemic events detected by diffusion-weighted MRI. Circ Cardiovasc Imaging 2012;5:376-82 CrossRef Medline

27. Chou MC, Tzeng WS, Chung HW, et al. T2-enhanced tensor diffusion trace-weighted image in the detection of hyper-acute cerebral infarction: comparison with isotropic diffusion-weighted image. Eur J Radiol 2010;74:e89-e94 CrossRef Medline

28. Samuels OB, Joseph GJ, Lynn MJ, et al. A standardized method for measuring intracranial arterial stenosis. AJNR Am J Neuroradiol 2000;21:643-46 Medline

29. Vittinghoff $\mathrm{E}, \mathrm{McCulloch} \mathrm{CE}$. Relaxing the rule of ten events per variable in logistic and Cox regression. Am J Epidemiol 2007;165:710-18 CrossRef Medline

30. Vittinghoff EG, Shiboski SC, McCulloch CE. Regression Methods in Biostatistics: Linear, Logistic, Survival, and Repeated Measures Models. Springer-Verlag; 2005:134

31. Royston P, Sauerbrei W. Bootstrap assessment of the stability of multivariate models. Stat Med 2009;9:547-70 CrossRef Medline

32. Sauerbrei W, Schumacher M. A bootstrap resampling procedure for model building: application to the Cox regression model. Stat Med 1992;11:2093-09 CrossRef Medline

33. Menon RS, Kidwell CS. Neuroimaging demonstration of evolving small vessel ischemic injury in cerebral amyloid angiopathy. Stroke 2009;40:e675-677-e677 CrossRef Medline

34. van Veluw SJ, Lauer A, Charidimou A, et al. Evolution of DWI lesions in cerebral amyloid angiopathy: evidence for ischemia. Neurology 2017;89:2136-42 CrossRef Medline

35. Boulouis G, Charidimou A, Auriel E, et al. Intracranial atherosclerosis and cerebral small vessel disease in intracerebral hemorrhage patients. J Neurol Sci 2016;369:324-29 CrossRef Medline

36. Honda K. Cerebral arterial occlusion did not promote the prevalence of cerebral amyloid angiopathy. J Alzheimers Dis 2016;54:26974 CrossRef Medline

37. Eng JA, Frosch MP, Choi K, et al. Clinical manifestations of cerebral amyloid angiopathy-related inflammation. Ann Neurol 2004;55:25056 CrossRef Medline

38. Sperling R, Salloway S, Brooks DJ, et al. Amyloid-related imaging abnormalities in patients with Alzheimer's disease treated with bapineuzumab: a retrospective analysis. Lancet Neurol 2012;11:24149 CrossRef Medline

39. Lassmann H. Pathogenic mechanisms associated with different clinical courses of multiple sclerosis. Front Immunol 2018;9:3116. CrossRef Medline 